\title{
Sébastien ABIS - Pour le futur de la Méditerranée : l'agriculture - Éditions L'Harmattan, Bibliothèque de l'iReMMO, Paris, 2012, 150 pages
}

\section{Thierry Pouch}

\section{(2) OpenEdition Journals}

Édition électronique

URL : http://journals.openedition.org/economierurale/4066

DOI : $10.4000 /$ economierurale.4066

ISSN : 2105-2581

Éditeur

Société Française d'Économie Rurale (SFER)

Édition imprimée

Date de publication : 15 octobre 2013

Pagination : 113-115

ISSN : 0013-0559

\section{Référence électronique}

Thierry Pouch, « Sébastien ABIS - Pour le futur de la Méditerranée : I'agriculture - Éditions L'Harmattan, Bibliothèque de l'iReMMO, Paris, 2012, 150 pages », Économie rurale [En ligne], 337 | septembreoctobre 2013, mis en ligne le 30 août 2013, consulté le 24 septembre 2020. URL : http://

journals.openedition.org/economierurale/4066; DOI : https://doi.org/10.4000/economierurale.4066

Ce document a été généré automatiquement le 24 septembre 2020.

(c) Tous droits réservés 


\title{
Sébastien ABIS - Pour le futur de la Méditerranée : l'agriculture - Éditions L'Harmattan, Bibliothèque de l'iReMMO, Paris, 2012, 150 pages
}

\author{
Thierry Pouch
}

1 Il est des livres dont on ne peut pas dire qu'ils se détournent d'une actualité brûlante. Ils collent au contraire à cette actualité économique, sociale autant que politique, et suggèrent des pistes pour essayer de résoudre des questions dont les enjeux dépassent le court terme. L'ouvrage de Sébastien Abis est de ceux-là. Il se penche sur une région du monde, les pays méditerranéens, lesquels sont entrés dans une phase de turbulence depuis quelques années. Le déclenchement de ce que l'on a communément appelé les "révolutions arabes » offre selon l'auteur une occasion de se pencher sur cette région, qui, si elle n'a pas réellement connu les "émeutes de la faim ", n'en est pas moins exposée à une forme d'insécurité alimentaire, lourde de menaces pour la stabilité politique des pays qui la compose, et par voie de conséquence, sur les pays voisins.

2 Une investigation qui tombe en quelque sorte bien et rend le propos des plus attractifs pour les lecteurs qui s'interrogent sur la situation interne autant qu'aux perspectives de sortie de crise. Ce court ouvrage, moins de 150 pages, n'en est pas moins un véritable instrument de réflexion, alliant la mise en perspective historique et un exercice de prospective, et traite essentiellement des Pays arabes méditerranéens (PAM). Il est issu d'une connaissance intime de la région, connaissance que met à profit Sébastien Abis pour inscrire les évènements du moment dans une perspective agricole et alimentaire.

3 L'ouvrage se compose de quatre parties, auxquelles s'ajoute une ultime partie plus prospective. La première, « dynamiques géopolitiques ", traite de manière synthétique de l'histoire de la région méditerranéenne, et avance des éléments d'analyse permettant de comprendre les origines des défaillances du processus de développement des pays arabes méditerranéens, qui les ont conduits à s'exposer de plus en plus à une vulnérabilité alimentaire, constituant l'une des dimensions du 
«printemps arabe ». Ce premier tour d'horizon invite le lecteur à penser la stratégie de l'Union européenne vis-à-vis de cet ensemble régional si proche. La deuxième partie de l'ouvrage, "dynamiques agricoles et rurales", insiste sur l'évolution du secteur agricole dans les PAM et en mesure les répercussions sur les productions et les conditions d'accès à l'alimentation des populations, dont certaines connaissent un taux d'accroissement élevé, à l'instar de l'Algérie. L'auteur montre bien en quoi cette dimension démographique joue un rôle de premier plan dans la dynamique alimentaire des PAM. Mais il invite au passage à bien examiner les ressources propres de ces pays. Or celles-ci font de plus en plus défaut et handicapent le processus même de développement agricole. Éléments chiffrés à l'appui, l'auteur indique que les facteurs de production - terre et eau plus spécifiquement - se sont raréfiés, induisant des tensions socio-politiques pour y avoir accès. Selon Sébastien Abis, la moitié de la population mondiale pauvre en eau - moins de 1000 mètres cubes par habitant et par an - se situe dans cette région. De plus, la région méditerranéenne devrait subir les effets du réchauffement climatique, lesquels devraient amplifier les contraintes pesant sur les productions.

4 Il s'ensuit que la production agricole est redevenue pour la plupart des pays une question stratégique. Mais les réponses qui sont apportées sont d'une grande variété, certains pays ayant relancé des dispositifs de politique agricole, tel le «Plan vert » au Maroc, la valorisation du potentiel agricole dans le Sud de l'Algérie, ou, solution plus radicale élaborée entre autres par le Qatar ou l'Arabie Saoudite, le recours à des locations ou des achats de terres cultivables.

5 La troisième partie du livre de Sébastien Abis, « dynamiques commerciales », couvre le champ des échanges commerciaux entre les PAM et le reste du monde. Après avoir resitué l'évolution des flux commerciaux globaux de produits agricoles et alimentaires, l'auteur revient sur la croissance des importations de ce type de produits des PAM depuis la fin de la décennie 1990. Il saisit en ce sens l'occasion que lui offrent les données statistiques pour suggérer au lecteur de prendre la pleine mesure de la dépendance alimentaire dans laquelle se trouvent désormais les PAM. Par surcroît, le solde agroalimentaire de leur balance commerciale n'a fait que se creuser depuis le milieu des années 2000, du fait de l'élévation des prix des denrées agricoles. C'est ce processus qui a un peu plus fragilisé, selon Sébastien Abis, des régimes politiques déjà fortement contestés. Il est rappelé que c'est dans les PAM que l'insécurité alimentaire a, avec l'Afrique subsaharienne, le plus augmenté entre 1990 et 2010.

6 Cette troisième partie est d'autant plus passionnante que l'auteur en examine les conséquences sur les stratégies géoéconomiques des pays producteurs et exportateurs de biens alimentaires. Pour répondre à une demande croissante exprimée par les PAM, les grands acteurs que sont le Brésil, les États-Unis, voire les pays de la Mer Noire, déploient des stratégies commerciales visant à conquérir ces marchés méditerranéens. Dans ce registre, l'agressivité commerciale du Brésil a été payante, puisque ses exportations sont passées en quelques années d'à peine 1 milliard de dollars en 2002 à plus de 5 milliards en 2011. C'est un véritable "corridor alimentaire " que le Brésil a mis en place avec les principales économies importatrices de la région, à commencer par l'Algérie, l'Egypte et le Maroc. En découle chez l'auteur une interrogation, qui apparaît être en réalité une incompréhension. Quelle est la position de l'UE dans ce système commercial? Quel positionnement entend-elle adopter face à la montée de 
pays émergents comme le Brésil, risquant d'amplifier les pertes de parts de marché sur cette région?

7 La quatrième partie apporte un éclairage de circonstance puisqu'elle traite des échanges céréaliers. C'est par le prisme de ces denrées de base (blé, orge, maïs) que l'auteur entend consolider sa démonstration. Les PAM sont non seulement installés dans une dépendance quantitative en céréales vis-à-vis de l'extérieur, mais elle se double d'un processus inflationniste consécutif de l'augmentation des prix agricoles depuis 2007. Au regard de ce qui a été traité par l'auteur dans les deux premières parties, la dépendance alimentaire risque de muter en "hyper dépendance ", laquelle est porteuse d'instabilité politique et sociale. C'est pourquoi il est si essentiel aux yeux de Sébastien Abis, de relancer le dialogue euro-méditerranéen, de donner une nouvelle impulsion au partenariat avec les économies du bassin méditerranéen. C'est l'objet de la cinquième et dernière partie du livre.

8 L'imposant raisonnement géopolitique entre alors en résonance avec la première partie, comme par un effet de bouclage. Au croisement de l'histoire, de l'économie, de l'agriculture et de la géopolitique, l'analyse se transforme en plaidoyer pour une présence affirmée de l'UE dans cette région. En matière agricole, il est dit que l'UE doit valoriser ses avantages comparatifs agricoles et alimentaires, et contribuer aux équilibres vitaux de cette région. La France étant le premier producteur de céréales de l'UE, elle peut en assumer le leadership.

9 L'ouvrage de Sébastien Abis est donc riche d'enseignements pour comprendre une région particulièrement instable et dont les perspectives d'autosuffisance alimentaire sont encore bien trop lointaines pour la laisser s'enfoncer dans une dépendance alimentaire dont on ne maîtrise pas totalement les conséquences à venir. L'exercice de prospective auquel s'est livré l'auteur mérite que l'on s'y arrête. L'originalité de l'approche retenue réside dans la combinaison d'une réflexion agricole, économique, et géopolitique. En d'autres termes, l'auteur a fait le pari, réussi, de la transdisciplinarité, pour lequel les observateurs, experts, à commencer par les économistes, sont souvent mal préparés.

10 Terminons cette note de lecture par un commentaire plus critique. Pour incontestables qu'ils soient, les mérites du livre de Sébastien Abis butent sur une limite. Le choix fait du périmètre arabe des pays méditerranéens pouvait en effet être élargi aux nations non arabes, tout du moins lorsque apparaît la dimension prospective du livre. La crise actuelle que traversent l'Espagne, la Grèce, l'Italie, désormais Chypre, se propage et a abouti à une instabilité politique certes encore plus ou moins maitrisée, mais réelle. Elle est porteuse d'une dégradation des conditions de production, y compris agricoles, d'une dégradation accélérée des comptes publics sous l'effet des récessions cumulées, et, in fine, d'une diminution du pouvoir d'achat des ménages. L'exercice prospectif conduit par l'auteur ne pouvait-il du coup s'accompagner d'un questionnement concernant l'émergence d'un second front de vulnérabilité alimentaire, au sein même de pays membres de l'UE ? Ce serait alors tout le Sud de la Méditerranée qui entrerait dans une instabilité sociopolitique, l'un des vecteurs en étant les contraintes pesant sur l'approvisionnement alimentaire.

11 On l'aura compris, l'ouvrage de Sébastien Abis livre des informations précieuses pour tout lecteur intéressé non seulement par l'agriculture mais aussi par sa dimension géopolitique. 


\section{AUTEUR}

\section{THIERRY POUCH}

APCA-DEAT, Laboratoire REGARDS, Université de Reims Champagne Ardenne 